\title{
Nightingale y la profesionalización de la enfermería en España
}

\section{Nightingale and the professionalization of nursing in Spain}

\section{Nightingale e a profissionalização da enfermagem na Espanha}

\section{Elena Santainés Borredá ${ }^{1}$ \& Ramón Camaño Puig ${ }^{2}$}

${ }^{1}$ Doctora por la Universidad de Valencia. Profesora Ayudante Doctor del Departamento de Enfermería. Facultat d’Infermeria i Podologia. Universidad de Valencia. E-mail: elena.santaines@uv.es. ORCID: https://orcid.org/0000-0001-7708-1134

${ }^{2}$ Doctor Europeo por Leeds Metropolitan University. Profesor Titular del Departamento de Enfermería. Facultat d’Infermeria i Podologia. Universidad de Valencia. E-mail: ramon.camano@uv.es. ORCID: https://orcid.org/0000-0002-8018-2232

Correspondencia:Departamento de Enfermería. Facultat d’Infermeria i Podologia. Universidad de Valencia. C/ Jaume Roig, s/n 46010-Valencia

Correo electrónico de contacto: elena.santaines@uv.es

Cómo citar este artículo: Santainés-Borreda, E. \& Camaño.Puig, R. (2021). Nightingale y la profesionalización de la enfermería en España. Cultura de los Cuidados. (Edición digital). 25 (No esp.). Recuperado de http://dx.doi.org/10.14198/cuid.2021.esp.11

Recibido:21/11/2020 Aceptado: 13/02/2021

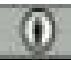

\section{RESUMEN}

La Historia de la Enfermería Contemporánea en España está siendo replanteada por múltiples historiadores, que luchan contra las distorsiones que han desvirtuado la identidad enfermera en la historia. Teniendo como punto de partida la Escuela de Enfermeras de Santa Isabel de Hungría (1896), fundada por Rubio y Galí y tomando como referencia la Nightingale Training School (1860) fundada por Nightingale; el presente estudio muestra algunas de las evidencias sobre cómo la profesionalización de la Enfermería en España siguió el mismo proceso que la enfermería en otros países. Analizando la información histórica desde la perspectiva de género, se muestra como el origen ideológico de la Enfermería 
Profesional en España coincide con el modelo de enseñanza enfermero promulgado por Nightingale. Un sistema de formación que llegó a nuestro país, por medio del cirujano Rubio y Galí, pero enmarcado en un modelo paternalista y una visión social de la mujer anclada al ámbito maternal y doméstico.

Palabras clave: Nightingale; enfermería contemporánea; perspectiva de género; Rubio y Galí.

\section{ABSTRACT}

The History of Contemporary Nursing in Spain is being rethought by multiple historians, who fight against the distortions that have distorted the nursing identity in history. Taking as a starting point the "Escuela de Enfermeras de Santa Isabel de Hungría” (1896), founded by Rubio y Galí and taking as a reference the Nightingale Training School (1860); This study shows some of the evidence on how the professionalization of Nursing in Spain followed the same process as nursing in other countries. Analyzing the historical information from the gender perspective, it is shown how the ideological origin of Professional Nursing in Spain coincides with the nursing teaching model promulgated by Nightingale. A training system that came to our country, through the surgeon Rubio y Galí, but framed in a paternalistic model and a social vision of women anchored in the maternal and domestic sphere.

Keywords: Nightingale; contemporary nursing; gender perspective; Rubio and Galí.
RESUMO

\section{A História da Enfermagem} Contemporânea na Espanha está sendo repensada por múltiplos historiadores, que lutam contra as distorções que distorceram a identidade da enfermagem na história. Tomando como ponto de partida a "Escuela de Enfermeras de Santa Isabel de Hungría” (1896), fundada por Rubio y Galí e tendo como referência a Nightingale Training School (1860) fundada por Nightingale; Este estudo mostra algumas evidências de como a profissionalização da Enfermagem na Espanha seguiu o mesmo processo da enfermagem em outros países. Analisando as informações históricas na perspectiva de gênero, mostra-se como a origem ideológica da Enfermagem Profissional na Espanha coincide com o modelo de ensino de enfermagem promulgado por Nightingale. Um sistema de formação que chegou ao nosso país, através do cirurgião Rubio y Galí, mas enquadrado num modelo paternalista e numa visão social da mulher ancorada na esfera materna e doméstica.

Palavras-chave: Nightingale; enfermagem contemporânea; perspectiva de gênero; Rubio e Galí. 
Revista científica de la Asociación de Historia y Antropología de los Cuidados (Universidad de Alicante)

\section{INTRODUCCIÓN}

La función identitaria de la historia de los cuidados ha generado y genera múltiples investigaciones que responden a la necesidad de combatir las distorsiones $\mathrm{y}$ omisiones que la han desvirtuado (Amezcua, González Iglesias y Solanas, 2018). Numerosos planteamientos que versan sobre cuestiones esenciales, entre las cuáles se encuentran ¿cuál es el origen de los cuidados profesionales en España? ¿Cómo se llevaban a cabo esos cuidados? ¿Quién dio los primeros pasos para la fundación de las primeras escuelas en la Enfermería contemporánea española? En el ámbito europeo, no cabe duda de que fue Nightingale, pero ¿y en España?

\section{DESARROLLO DEL TEMA}

\section{Florence Nightingale (1820-1910)}

Nightingale, conocida internacionalmente por su labor en la Guerra de Crimea (1854-56), configuró un plan administrativo, y desarrolló procedimientos para el tratamiento de los datos y su representación gráfica que permitiesen conocer cómo iba evolucionando la situación con las reformas e intervenciones propuestas (Woodham Smith, 1957). Consecuentemente, desarrolló un plan de formación de enfermeras con el "Fondo Nightingale” e inauguró, en 1860, La Nightingale Training School for Nurses, como una institución educativa independiente. A nivel teórico Nightingale empleó las bases de su obra, Notes on nursing. What is it, and what it is not (1859). Paralelamente, en 1861, creó una Escuela Práctica para Parteras, cooperando con autoridades del King's College de Londres, centro donde estudió Lister y estuvo W. Fergusson; y desarrolló una sala de maternidad. Además de la asistencia a enfermos, Nightingale, seguidora del higienismo, consideraba prioritaria la atención de “la cuestión social”, relacionada con la higiene urbanística de la ciudad. Consiguió la elevación de los índices de salubridad y la reducción de las tasas de mortalidad materno-infantil introduciendo modificaciones en el rol de la mujer en el ámbito doméstico (Hernández Conesa, 1995; González Canalejo, 2010).

La influencia del Sistema de Nightingale originó la creación de una gran cantidad de escuelas, que siguieron su modelo de enseñanza, tanto en las Islas Británicas, como Irlanda, Estados Unidos, Dinamarca, Suecia, Australia, Finlandia, Roma, China, Beirut, Buenos Aires y distintas zonas de Sudáfrica. Sin embargo, España no aparece entre los países subsidiarios del sistema de enseñanza promovido por Nightingale (Santainés, Faus, Camaño, Sarturi, 2012). Las primeras referencias a Nightingale en España llegaron a inicios del siglo XX, en forma de escritos que rememoraban la labor de una mujer en el campo de la ciencia (Feminal, 1911; Matters, 1916). 


\section{Federico Rubio y Galí (1827-1902)}

Rubio y Galí, médico cirujano, tuvo una vida vinculada no solo al campo de las Ciencias de la Salud, sino también a la esfera política, motivo por el cual estuvo en Inglaterra como embajador del reino de España, entre 1860-1870, cargo que no llegó a ejercer, pues dicho régimen español no fue reconocido por el gobierno británico (Rubio y Galí, 1977; Campos Marín, 2004). Durante este tiempo permaneció exiliado, en Inglaterra, coincidiendo en el tiempo con las reformas que acometió Nightingale de la enfermería. Las consideraciones de Hernández Sandoica (1984), revelan que la mayor parte de su estancia la dedicó al estudio. Suárez Ávila en el prólogo de la obra Memorias de Rubio y Galí (1977), nos decía que, mientras estuvo en Londres, asistió a los cursos del célebre cirujano William Fergusson (1808-1877), seguidor de los principios antisépticos de Lister y miembro del King’s College de Londres.

A su regreso, en 1880, constituyó el Instituto de Terapéutica Operatoria (ITO), en el Hospital de la Princesa en Madrid. En 1896, fundó la primera escuela de enfermeras en España, la Escuela de Enfermeras de Santa Isabel de Hungría, constituida en un ámbito hospitalario público dominado por la reforma de la Beneficencia y conviviendo con titulaciones como los practicantes. Además, consideró la continuidad de la tradición religiosa, conservando la visión social de la mujer dentro del ámbito doméstico y maternal (Marset Campos \& Sáez Gómez, 2003; Campos Marín, 2004). La formación recibida en la escuela se basaba en nociones muy similares a las divulgadas por Nightingale en su Escuela de Enfermeras. Con estos principios fueron sucediendo promociones de enfermeras, siendo la última la de 1932, previa a la Guerra Civil Española (Álvarez Nebreda, 2007), durante la cual se perdió gran parte de la documentación relacionada con esta escuela. Y es precisamente en este punto donde surge la cuestión acerca de dónde recogió el cirujano su modelo de enseñanza y las ideas higienistas que transmite en su escuela. La historia plantea cierta similitud con los postulados de Nightingale, veamos si la documentación histórica nos ayuda a perfilar dicha proximidad.

\section{Filosofia enfermera de Nightingale en Rubio y Galí}

Los documentos escritos por Rubio y Galí y Nightingale nos permiten realizar una aproximación al origen ideológico del modelo de enfermería propuesto por el cirujano (Nightingale, 1859, 2004; Rubio y Galí, 1881-1885). El análisis del contenido de los escritos de ambos autores, desde una perspectiva cualitativa y cuantitativa, ha sido realizado mediante el uso del software WordSmith 6.0, programa que nos permite el análisis de concordancias textuales. En términos cualitativos, en la Tabla 1 se 
Revista científica de la Asociación de Historia y Antropología de los Cuidados (Universidad de Alicante)

presentan ciertas semejanzas ideológicas y podredumbre causó en sus heridos” (Rubio coincidencias entre los planteamientos de y Galí, et al., 1882, pp. 132).

Rubio y Galí y las materias que Nightingale desarrolla en Notes on Nursing (Santainés, 2019).

Directamente, no es demostrable que, entre sus recuerdos, se incluya su conocimiento relacionado con la intervención de Nightingale como enfermera

Tabla 1. Semejanzas ideológicas sobre los cuidados enfermeros en los escritos de Nightingale y Rubio y Galí.

\begin{tabular}{ll} 
Nightingale (Notes on Nursing, 1859) & Reseñas de Rubio y Galí (1881-85) \\
\hline $\begin{array}{l}\text { Administración y cuidado de los } \\
\text { pequeños detalles }\end{array}$ & $\begin{array}{l}\text { Atención a los pequeños detalles. Variabilidad y } \\
\text { especificidad en cada caso. La importancia de la rapidez e } \\
\text { idoneidad en la atención }\end{array}$ \\
Alimentación & $\begin{array}{l}\text { Respetar la puntualidad y no obligar al enfermo. } \\
\text { Alimentación r/c los pobres y débiles. }\end{array}$ \\
Clases de Alimentos & $\begin{array}{l}\text { Respetar el gusto y la costumbre. Atender al estado } \\
\text { nutritivo del enfermo. La importancia de la asimilación } \\
\text { gástrica. Dieta a base de carne, lácteos, vino. }\end{array}$ \\
Charlatanería, esperanzas y consejos & $\begin{array}{l}\text { Influencia de las visitas de amigos y familiares. Informar } \\
\text { sobre la verdad al enfermo }\end{array}$
\end{tabular}

La Cama y Ropas de cama

Arreglo, limpieza e higiene de las ropas de cama. Atención al peso sobre el paciente

La luz

Beneficios de la luz natural en el enfermo

Limpieza habitaciones/ paredes

Limpieza e higiene de las habitaciones y paredes

Limpieza/higiene personal

Limpieza e higiene de las salas quirúrgicas, importancia de la experiencia y la práctica en la enseñanza Higiene continua del enfermo, lavado relacionado con la Temperatura Corporal, cambio de ropas de ropa

Conocimiento de la verdad con la observación, peligrosidad

Observación del Enfermo de la imaginación, atender al aspecto del enfermo, observar el entorno y las condiciones en que vive

Ruido Evitar ruidos innecesarios. La importancia de la ligereza y suavidad en el cuidado. Atender al descanso/sueño. La lectura.

Salubridad de las casas La luz como causa de la escrófula. Prevención de la infección. Cuidados continuos. Contagio por aire. Enfermedad como noción categórica

Ventilación y Calefacción Aire puro/natural, ventilación de la sala del enfermo, problemas relacionados con la humedad

Contextualizando estas semejanzas mediante un análisis del discurso se rescata una afirmación de Rubio y Galí, en la que reconoce: "Nosotros leímos algo de la guerra de Crimea; conservamos algunos recuerdos de los estragos que la en dicho conflicto bélico. No obstante, parece razonable creer que tuviera algún conocimiento del impacto que tuvo la labor de Nightingale en dicho contexto dado el impacto social que tuvieron sus acciones. Si bien es cierto que Rubio y Galí, como cirujano, se interesó más por el estado 
clínico, tratamiento y evolución de los enfermos quirúrgicos; no se debe obviar que Nightingale tuvo un papel protagonista en los cuidados relacionados con la higiene del paciente quirúrgico. Si, además, recordamos que el cirujano estuvo durante la década de 1860-70 en Londres, y consideramos el número de paralelismos conceptuales expuestos de forma resumida en la Tabla 1 , todo induce a pensar que tuvo interés por conocer la Escuela de Nightingale e incluso su obra más conocida, Notes on nursing.

Las coincidencias reflejadas en la Tabla 1 se mantienen en otras fuentes documentales primarias, en donde se ratifica la influencia de Nightingale sobre el modus operandi de Rubio y Galí; no solo en aspectos relativos a la ideología enfermera, sino en el ámbito sanitario en general. En publicaciones previas (Santainés, Faus y Camaño, 2016), se han realizado consideraciones acerca de la preocupación que Rubio y Galí junto con Olavide, tienen por la aireación de la habitación del enfermo. En una de sus publicaciones, corroboran la importancia que le daban a la atmósfera de las habitaciones de los enfermos. Proceden a examinar el aire de la buhardilla a la que habían sido trasladados los enfermos de la sala de Martín de Pedro del Hospital General, de Madrid, por causa del empeoramiento de los enfermos ingresados (Rubio y Galí, 1872):

"[...] el vapor de agua que hemos examinado bajo una forma líquida encierra sustancias nocivas para la respiración de los enfermos y presupone una atmósfera de todo punto antihigiénica y que conviene sanear, poniendo en práctica y vigor las leyes higiénicas en mal hora quebrantadas" (Rubio y Galí, 1872, pp. 436-437).

Si hablamos, por ejemplo, del lecho del enfermo, Rubio y Galí cita la "cama como medio terapéutico”. Nightingale no lo hizo textualmente en su obra, pero destinar un capítulo completo a este aspecto, denota lo importante y primordial que era para ella la cama y sus ropas en el cuidado, en términos de ventilación, composición, colocación y disposición. Si se hace referencia a la estructura de las salas hospitalarias, el médico indica que las paredes del ITO son de yeso, coincidiendo con las palabras de Nightingale en una de sus publicaciones: Notes on Hospitals (1863). La enfermera no solo afirma cómo la mayoría de los hospitales empleaban paredes enyesadas, sino que además enumera las ventajas e inconvenientes, así como la forma de solucionar o disminuir los problemas que de esas paredes porosas se pueden obtener. A razón de la estructura y organización de la institución, Rubio y Galí indica que el:

"ITO comenzó su organización, estableciéndose la Clínica, compuesta de dos salas de á 20 camas, una para hombres y otra para mujeres" (Rubio y Galí, 1881, pp.1).

Y, previamente, Nightingale en su libro Notes on Hospitals ya hizo mención a ello: "[...] the best size of wards for ensuring the two condi-tions of health and facility of administration and discipline, is from 20 to 32 sick" (Nightingale, 1863, pp. $62)$. De hecho, en Notes on Hospitals expone el estado en el que se hallaban los hospitales contemporáneos, así como las nociones de ventilación, limpieza e higiene necesarias para el establecimiento de una institución sanitaria. Por ello, Nightingale recibió proyectos desde Portugal, Prusia y Holanda, entre otros, por lo que es factible plantearnos que Rubio y Galí acogió estas ideas de Nightingale para la construcción y diseño del ITO; lo que constituye una buena pregunta de partida para una investigación relacionada con aspectos de estructura arquitectónica de los hospitales.

Una curiosidad que se desprende del análisis de los escritos de Rubio y Galí es que no hace mención del lavado de manos. Sin embargo, es posible localizar citas que 
afirman de la importancia que se le daba a esta técnica como: "[...] el cirujano [Rubio y Galí] y sus ayudantes visten blusas y delantales de lienzo y someten sus manos a un detenido lavado con agua y jabón primero, y posteriormente de sublimado $y$ alcohol" (López-Ríos Fernández, 2003 pp. 177).

Nightingale reconoce la importancia del lavado de manos aludiendo a la necesidad de un frecuente lavado de manos con agua y jabón:

"Every nurse ought to be careful to wash her hands very frequently during the day. If her face too, so much the better [...] if a little spirit be added to it, it will be more effectual [...] To wash with soap and soft water" (Nightingale, 1859).

Este hecho, nos hace pensar en la importancia que debería atribuirse a Nightingale como una de las primeras en el campo de la Antisepsia, además de Lister cuyas repercusiones sobre Rubio y Galí fueron temporalmente más tardías, como se puede apreciar en la obra de Juan Riera (1973). Paralelismos que nos hacen considerar la posibilidad de que el conocimiento de Rubio y Galí sobre lo acaecido en la Guerra de Crimea tuviera su origen, aparte de su experiencia en la sociedad victoriana de la época, en que pudiera haber habido algún contacto con Nightingale; dado que, entre las relaciones profesionales del cirujano en Londres durante su exilio, estuvieron figuras como Fergusson, vinculado con el St Thomas' Hospital y la Nightingale Training School, e igualmente visitó el King's College de Londres, vinculado con Lister. Cuestiones que incrementan la posibilidad de que se produjese un encuentro entre ambos, lo que puede ser analizado mediante la Teoría de los Seis Grados de Separación, propuesta por Karinthy (1929); una hipótesis que intenta probar que cualquier persona en la Tierra puede estar conectado a cualquier otra persona del planeta a través de una cadena de conocidos que no tiene más de cinco intermediarios, lo que nos permite valorar la posibilidad de que Nightingale y Rubio y Galí estuviesen, de alguna manera, conectados; a partir de cinco personas interpuestas para verificar dicha hipótesis (Watts, 2005). La demostración mediante contactos sociales personificados requiere de nuevas indagaciones vinculadas al estudio pormenorizado de sus trayectorias personales y profesionales.

Llegados a este punto es factible encaminarse hacia la convicción de que la ideología enfermera de Nightingale también trascendió en España por la mediación de Rubio y Galí. Corroboran esta afirmación, ciertas declaraciones de compañeros del cirujano que escribieron en la misma revista que Rubio y Galí fundó. Pulido Martín (1906, pp. 158) se expresaba así:

"Dependencia del Hospital de Londres es la gran Escuela de nurses (enfermeras), capaz para quinientas [...] No cabe duda de que las nurses son uno de los factores del adelanto de nuestra ciencia en aquel país, y ese cuerpo de tan eficaces auxiliares médicos es copiado en todas las naciones, una de ellas la nuestra, donde el genio del inmortal Don Federico Rubio hizo mucho inspirándose en ella".

Y Yagüe (1924, pp.131), nos indicaba que los fundamentos del progreso que constituyó la Escuela de Santa Isabel de Hungría se pueden encontrar en Inglaterra: "Don Federico [...] creando [...] la Escuela de enfermeras de Santa Isabel de Hungría, aneja al Instituto de Terapéutica Operatoria. Sus fundamentos pueden encontrarse en Inglaterra, mas es el iniciador de este progreso sanitario en España” 
Estas declaraciones nos llevan a pensar si los autores hacían referencia a la Nightingale Training School. Se han presentado evidencias para dar una respuesta afirmativa; pero se cuestiona por qué no se nombra a Nightingale, así como a su escuela, pues queda demostrado una conciencia asimilada de los conocimientos y postulados enfermeros de Nightingale por parte de Rubio y Galí. Lo que nos lleva a pensar en una posible ocultación del reconocimiento de su labor enfermera.

\section{Principios sanitarios de Nightingale en Rubio y Galí}

Recordemos que Rubio y Galí, como intelectual krausista de la Institución Libre de Enseñanza (ILE), pretendía educar a la mujer en aspectos relacionados con la corriente higienista predominante de la época, movido por la necesidad de paliar el problema de la demografía. Por su parte, Nightingale se ocupó de resolver el más elemental de los problemas: la higiene del ambiente y del individuo, mediante métodos preventivos e instrucción en Salud Pública. No sería erróneo afirmar que Nightingale fue una de las predecesoras en la lucha por la prevención de la infección. Por lo que, de alguna forma se anticipó mediante la intuición, desde la práctica empírica, a los científicos Pasteur y Koch, siendo consciente de los principios coetáneos de la cura aséptica de Lister.

Desde la Salud Pública en el libro $L a$ mujer gaditana (1902), Rubio y Galí describe cómo interviene la naturaleza misma sobre el ser humano, distinguiendo el medio interno del externo, y dándole valor a elementos claves para el cuidado, descritos ya por Nightingale: ventilación $y$ calefacción, salubridad de las casas, ruido, alimentación, luz, limpieza, etc.

$\mathrm{S}$

iguiendo con La mujer gaditana, encontramos esa preocupación de Rubio y
Galí en lo que él llamaba "inadvertidos lavaderos domésticos”; dedica unas páginas a describir cómo se realiza la colada y los cambios de ropa en los domicilios de las familias gaditanas. Este apunte es quizás otra similitud ligada a los principios de Nightingale. Si recordamos la Guerra de Crimea, fue Nightingale quien ideó una lavandería alquilando una casa turca en Scutari, en donde trabajaban las mujeres de los soldados.

Adicionalmente, indagamos en más fuentes para valorar si Nightingale trascendió en otros campos. Fue autora de una sección del Quain's Dictionary of Medicine (1882a, b), en el cual expuso el concepto de enfermera y de su formación, así como los cuidados para enfermedades concretas. Una intervención en una publicación médica que demuestra cómo estaba considerada dentro del colectivo médico. Y, sin embargo, no es la única. En 1871, publicó Introductory Notes on Lyingin Institutions, junto con el Dr. Tutherland del King's College Hospital de Londres, en donde describe los resultados obtenidos tras su investigación sobre las causas de la alta mortalidad en las instituciones de maternidad y las salas de hospital, frente a los partos en domicilios particulares (Nightingale, 1871). Ella misma proponía que el uso de las pequeñas habitaciones que mantenían separados a los enfermos suponía una medida para subsanar el alto porcentaje de mortalidad en los partos, además de la aplicación de las correspondientes medidas higiénicas. Se podría afirmar que esta publicación, pudiese ser una de las más relacionadas con las ideas promulgadas por Ignac Semmelweis.

Nightingale aplicó también sus principios sanitarios en el ámbito militar, con Notes on matters affecting the health efficiency and hospital administration of the British Army founded (1858); una obra con estadísticas y un exhaustivo plan de estudios 
organizado y gestionado para la educación de los médicos militares. Médicos militares y cirujanos de diferentes países conocieron sus aportaciones en este campo, por lo que, Rubio y Galí, pudiese ser uno más dentro del círculo de profesionales a los que llegaron sus postulados.

En 1858 fue invitada a formar parte como miembro de la Royal Statistical Society de Londres, impulsora de la elaboración de un registro estadístico que permitiese, mediante tablas y gráficos, la toma de decisiones clínicas más efectiva. Con su trabajo consiguió que los distintos hospitales de Londres (entre ellos el King's College Hospital y St. Thomas' Hospital) adoptaran un sistema uniforme de registro de los enfermos y publicaran anualmente sus estadísticas. Del mismo modo, en las fuentes primarias de Rubio y Galí (1881-85) se observan tablas de registro estadístico, relacionadas con el número de intervenciones quirúrgicas atendiendo a los casos clínicos, sexo e incluso su evolución posterior.

\section{Nightingale en España desde la perspectiva de género}

Este trabajo requiere de un último análisis incorporando la perspectiva de género y de clase. Pues a través de éste se explica, cómo se ha podido velar la realidad de los orígenes ideológicos de la Enfermería contemporánea en España (Cano-Caballero, 2004 y Chamizo, 2004), en relación con la posición de la profesión enfermera basada en la perspectiva de género. Diversos autores, entre ellos Narváez, Martínez \& Pérez (2010) sostienen que el ideal de feminidad de la época reflejaba la exclusión de la mujer en la vida pública, quedando diferentes sectores exclusivamente en manos del hombre; justificando dicha afirmación en la mera diferencia biológica, que derivaban a hombres y mujeres hacia roles sociales distintos. Como era obvio, la posición de Nightingale no encuadraba en dichos cánones. Amaro Cano (1991), ya lo decía insistiendo en como la mera condición de ser mujer ya impedía la paridad social con el hombre.

Este contexto era perfectamente extrapolable a la sociedad española de finales del siglo XIX y principios del XX, donde podría incluirse el pensamiento expuesto por Rubio y Galí en su obra $L a$ mujer Gaditana:

"La Naturaleza tiene sus leyes. La mujer nació para madre: para providencia, en caso necesario: para directora de su casa: para educadora ejemplar de hijos y marido; para distribuidora económica del producto del trabajo del hombre; para adorno, placeres, caricias, alegría y amor de la familia. ¿Parece poco? ¿Hay quien piense que resulta rebajada en la importancia de funciones comparativamente al hombre? Pues sostengo y pruebo que en todos sentidos son sus funciones tan altas, cuando menos” (Rubio y Galí, 1902, pp. 188).

A lo que añade:

" $Y$ vamos al aspecto aristocrático de la cuestión, que también las cuestiones socialistas tienen su aristocracia. ¿Por qué no ha de ser la mujer médica, abogada, ingeniera, catedrática, militar, diputada ó ministra? Por mí, que lo sea en buena hora [...] pero sería muy contra mi gusto y consejo que hiciesen profesión de nada de esto. Al menos, si tuviese que elegir esposa, huiría como de la peste de semejantes profesionales [...]" (Rubio y Galí, 1902, pp. 191-192).

A la mujer española se le atribuyen todas las tareas domésticas, y se las eleva al calificativo de iguales en importancia como las responsabilidades del hombre, lo que no es más que otra muestra de esa evidente distinción de género, cada cual en su lugar. 
En relación con la mujer enfermera; Rubio y Galí en su carta a las enfermeras titulada Introducción a las Instrucciones para las enfermeras internas (La redacción, 1927) dice que las mujeres prefieren escoger la vida de enfermeras a cualquier riqueza, honor o placer, considerando que los lavaderos y las cocinas de los hospitales son como tronos para que se sientan como reinas, pero viviendo como penitentes. Al margen de dichas afirmaciones, consideramos que debemos tomar conciencia de cuán difícil podría resultar para Rubio y Galí asimilar la existencia e inclusión de la mujer en igualdad de derechos con los hombres, concretamente, en lo que respecta a la ideología de una mujer enfermera británica. Lo cual no es una cuestión sencilla, sino de alto nivel de complejidad, por lo que no lo tenía nada fácil para el contexto en el que vivió.

Diferentes referencias en periódicos de la época como El Gráfico y Heraldo de Madrid nos aportan nuevas afirmaciones sobre las enfermeras de la escuela Rubio y Galí. Por ejemplo, el firmante del siguiente escrito que se identificó como "Compañero de Caza de Don Federico” (1904, pp. 7):

"Estás enfermeras son dignas del aprecio de todos, por su misión humanitaria: no tienen sueldo ni esperan nada; hacen el bien por el bien mismo. [...] Recluidas en el establecimiento [...] con humildes alpargatas abiertas y las piernas desnudas, bajo su traje de rayadillo, limpias como nadie [...] son él complemento de los difíciles trabajos operatorios llevados á efecto por el cultísimo profesorado del Instituto".

Saint-Aubin (1915, pp. 1) nos describe las duras condiciones de vida en la Escuela, así como el nivel de sumisión que se esperaba de las mujeres aspirantes:

"La más estrecha regla conventual no se impone con mayor dureza a las enclaustradas que el reglamento a las enfermeras del Instituto. Han de obedecer sin réplica las órdenes que corresponden a los deberes de su penoso cargo. No han de entablar conversaciones, limitándose a contestar sí o no [...] Han de abstenerse de visitas, salidas del Hospital, relaciones exteriores, correspondencias, noviazgos y tocar moneda [...]"

Rubio y Galí fundó una escuela de enfermeras que trataba de mantener una tradición religiosa basada en la no remuneración, la obediencia y la supeditación al hombre, con el mantenimiento de una fuerte estructura jerárquica basada en la historia natural. Con este tipo de descripciones, es inevitable pensar en esa fuerte distinción de género, y más todavía si se consideraba que las alumnas estaban viviendo como "penitentes", sin visitas, prohibición de cualquier relación con el exterior. En contraposición se hablaba de los profesores como "cultísimos"; por lo que hombre y mujer no estaban en la misma escala de valores sociales.

Podríamos encontrar muchos ejemplos más, en fragmentos localizados en la revista que el mismo cirujano fundó, la Revista iberoamericana de ciencias médicas y en discursos de los propios compañeros de Rubio y Galí; en otros periódicos de la época; en sus propios biógrafos, por ejemplo, la conocida biografía publicada por Álvarez-Sierra, quien escribió El Doctor D. Federico Rubio. Vida y obra de un cirujano genial (1947), escritos que son analizados con detalle en la obra recientemente publicada por Santainés (2019).

El análisis que realiza Hernández Sandoica (1984) sobre el cirujano plantea que Rubio y Galí vivía bajo una doble condición, era hombre y médico. Por el contrario, Nightingale era mujer y enfermera. La medicina ha sido la profesión que más se opuso al interés y la lucha 
Revista científica de la Asociación de Historia y Antropología de los Cuidados (Universidad de Alicante)

femenina a favor de la profesionalización de la enfermería. Por todo ello, abogamos por historiar los hechos y la investigación que de manera rigurosa realice aportaciones fuera de la "empresa del conocimiento mitificado”. Hernández (2010) afirma que se continúan escribiendo historias incompletas, parciales y paralelas, que tratan biografías, en las que se exaltan unos aspectos e ignoran otros. La Historia requiere de una concepción testimonial cambiante, en la que hay y habrá errores, acompañados siempre de aciertos en curso. El cumplimiento o no de nuestras expectativas depende de nuestra conciencia como enfermeras y nuestra lucha por conocer cuál ha sido el verdadero camino que nos ha permitido estar donde estamos. Desarrollamos y fundamentamos nuestra profesión mediante procesos de razonamiento reflexivos, lógicos y críticos. En esto se deberá de basar nuestra actividad y nuestro futuro, caminamos construyendo nuestra historia, la historia ya escrita muchas veces supeditada y determinada por su anclaje social, y, por ende, de género.

\section{CONCLUSIONES}

Con el análisis expuesto se podría concluir que Nightingale, contribuyó a la fundación de la primera escuela de Enfermería en España. Siendo Rubio y Galí un impulsor e innovador de los cuidados enfermeros y transmisor de un conjunto de ideologías relacionadas con la figura de la enfermera como profesión.

El modelo de formación de Rubio y Galí respondía a un modelo paternalista; la mujer fue buscada para recluirla al ámbito sanitario hospitalario; continuando la tradición religiosa de la mujer en lo vinculado a lo religiosos $\mathrm{y}$ al cuidado no remunerado. A través de esta visión de género y el análisis social hemos encontrado ciertas claves de invisibilidad de la enfermería a veces por un posible ocultamiento que ha impedido su reconocimiento.

Nightingale fue una de las predecesoras de la lucha en la prevención de la infección, desde la perspectiva práctica empírica. Por lo que, de alguna forma se anticipó a los descubrimientos de Pasteur y Koch, siendo consciente de los principios coetáneos de la cura aséptica de Lister. Todos estos argumentos constituyen razones para plantear una necesidad o conveniencia hacia investigaciones que incorporen la perspectiva de género, a corrientes de pensamiento, como por ejemplo el higienismo. Adicionalmente, se proponen como nuevas líneas de investigación, la aplicación de la Teoría de los Seis Grados de Separación vinculando la posibilidad de un conocimiento entre ambos, respondiendo a los contactos profesionales comunes entre ellos.

El análisis del contexto histórico nos ha permitido valorar las aportaciones de quienes lucharon por la Enfermería, no solo desde una perspectivita profesional, sino sobre todo desde una visión de género y clase. Por ello, reiteramos que nunca podemos dejar de aprender de lo escrito, puesto que las "verdades" que hemos leído y estudiado en los libros de historia de la Enfermería en España pueden plantear siempre "nuevas verdades".

\section{BIBLIOGRAFÍA}

Álvarez Nebreda, C. (2007). Historia de la Enfermería: ocurrió hace cien años. Tribuna Sanitaria, (206), 19-20.

Álvarez-Sierra, J. (1947). El Doctor D. Federico Rubio. Vida y obra de un cirujano genial. Madrid: Editora Nacional.

Amaro Cano, M.C. (1991). Algunas consideraciones sobre la personalidad histórica de Florence Nightingale. Revista Cubana de Enfermería, 7 (1), 59-66.

Amezcua, M; González Iglesias, M.E.; 
Antón Solanas, I. (2018). Distorsiones en la Historia de la Enfermería, un consenso virtual. Temperamentvm; 14. Recuperado de: http://ciberindex.com/c/t/e33002.

Campos Marín, R. (2004). Curar y gobernar. Medicina y liberalismo en la España del siglo XIX. Monlau, Rubio y Giné. Tres Cantos (Madrid): Nivola Libros y Ediciones, S.L.

Cano-Caballero Gálvez, M. D. (2004). Enfermería y género tiempo de reflexión para el cambio. Index de Enfermería, 13 (46), 34-39.

Chamizo Vega, C. (2004). La perspectiva de género en Enfermería, comentarios $\mathrm{y}$ reflexiones. Index de Enfermería, 13 (46), 40-44.

Compañero de Caza de Don Federico (1904). Don Federico Rubio. Instituto Rubio. Las enfermeras. El Gráfico, (80), 78.

Feminal (1911). La dona i les seues obres de beneficiència en el estranger. Feminal, (50): 8-9.

González Canalejo, C. (2010). Mujeres Sanitarias y derechos de ciudadanía en España (1868-1936). En C. González Canalejo, F. Martínez López (Eds.), La transformación de la Enfermería. Nuevas miradas para la historia (pp. 163-181). Granada: Editorial Comares.

Hernández Conesa, J. (1995). Historia de la Enfermería. Un análisis histórico de los cuidados de enfermería. Madrid: McGrawHill Interamericana.

Hernández Martín, F. (2010). Balance de dos décadas (1988- 2008): contribución de la historia de la enfermería a la historiografía española. En C. González Canalejo, F. Martínez López (Eds.), La transformación de la Enfermería. Nuevas miradas para la historia (pp. 499-510). Granada: Editorial Comares.

Hernández Sandoica, E. (1984). Reforma social e iniciativa privada en un médico español del XIX: Federico Rubio y Galí y su concepción de la sociedad. Asclepio, (36), 325-346.
La redacción. (1927). Principales datos biográficos del Dr. D.Federico Rubio y Galí. Revista ibero-americana de ciencias médicas, II (21), 23-24.

López-Ríos Fernández, F. (2003). El legado quirúrgico de Federico Rubio. En J. L. Carrillo Martos (Ed.), Medicina, Estado y Sociedad en la España de la segunda mitad del siglo XIX (pp. 141-182). El Puerto de Santa María: Ayuntamiento de El Puerto de Santa María y Asociación para la Formación, Investigación y Asistencia Médica de Andalucía "Federico Rubio".

Marset Campos, P., Sáez Gómez, J. M. (2003). Medicina, Estadoy Sociedad en la España de la segunda mitad del siglo XIX. En J.L. Carrillo Martos (Ed.), Medicina y Sociedad en la España de la segunda mitad del siglo XIX: una aproximación a la obra de Federico Rubio y Galí (1827-1902) (pp. 1-34). El Puerto de Santa María: Ayuntamiento de El Puerto de Santa María y Asociación para la Formación, Investigación y Asistencia Médica de Andalucía "Federico Rubio".

Matters, M. (1916) Florence Nightingale. "La Dama De La Llàntia". Quaderns d'Estudi. Revista Especialment Dedicada a Mestres i Professors, II (5), 336-341.

Narváez Traverso, A., Martínez Galíano, J. M., Pérez Martín, B. (2010). Revisitando a Florence Nightingale desde una perspectiva de género. Index de Enfermería, 19 (4), 299302.

Nightingale, F (1871). Introductory Notes on Lying-in Institutions. London: Longmans, Green and Co.

Nightingale, F. (1858). Notes on matters affecting the health efficiency and hospital administration of the British Army founded. London: Harrison and Sons, St. Martin's Lane W.C.

Nightingale, F. (1859). Notes on nursing. What is it, and what it is not. New York: D. Appleton and Company.

Nightingale, F. (1863). Notes on Hospitals (3rd Ed). London: Longman, Green, Longman, Roberts and Green. 
Revista científica de la Asociación de Historia y Antropología de los Cuidados (Universidad de Alicante)

Nightingale, F. (1882a). Nurses, training of. En R. Quain (Ed.), A dictionary of medicine including general pathology, general therapeutics, hygiene, and the diseases peculiar to women and children (pp. 10381043). New York: D. Appleton and company.

Nightingale, F. (1882b). Nursing the sick. En R. Quain (Ed.), A dictionary of medicine including general pathology, general therapeutics, hygiene, and the diseases peculiar to women and children (pp. 10431049). New York: D. Appleton and company.

Nightingale, F. (2004). Notas de enfermería: qué es y que no es. (J. Castro Vizoso Trans.). Elsevier MASSON.

Pulido Martín, A. (1906). Los Hospitales de Londres. Revista ibero-americana de ciencias médicas, XV (XXIX), 155-160.

Riera, J. (1973). La introducción en España del método antiséptico de Lister. Valladolid: Seminario de Historia de la Medicina.

Rubio y Galí, F. (1872). Sobre el examen microscópico del vapor atmosférico de la enfermería del doctor Martín de Pedro en el Hospital General. El Siglo Médico, (XIX), 404-437.

Rubio y Galí, F. (1902). La mujer gaditana. Apuntes de economía social. Madrid: Establecimiento tipográfico de Idamor Moreno.

Rubio y Galí, F. (1977). Memorias (Fragmentos). Cádiz: Casa de la Cultura Puerto de Santa María.

Rubio-Galí, F. (1881). Reseña del primer ejercicio del Instituto de Terapéutica Operatoria del Hospital de la Princesa. Enrique Teodoro Imp.

Rubio-Galí, F., Ariza Espejo, R., \& Buisen, S. (1882). Reseña del segundo ejercicio del Instituto de Terapéutica Operatoria del Hospital de la Princesa. Enrique Teodoro Imp.

Rubio-Galí, F., Ariza Espejo, R., Buisen, S., \& Gutiérrez, E. (1883). Reseña del tercer ejercicio del Instituto de Terapéutica
Operatoria del Hospital de la Princesa.

Enrique Teodoro Imp.

Rubio-Galí, F., Ariza Espejo, R., Buisen, S., Gutiérrez, E., \& Martínez Ángel, A. (1884). Reseña del cuarto ejercicio del Instituto de Terapéutica Operatoria del Hospital de la Princesa. Enrique Teodoro Imp.

Rubio-Galí, F., Ariza Espejo, R., Buisen, S., Gutiérrez, E., Martínez Ángel, A., \& Zabala, J. (1885). Reseña del quinto ejercicio del Instituto de Terapéutica Operatoria del Hospital de la Princesa. Enrique Teodoro Imp.

Saint-Aubin, A. (1915). Los hospitales. Instituto Rubio. Un poco de historia. Heraldo de Madrid, XXVI (8958), 1-2.

Santainés Borredá, E. (2019). Florence Nightingale en la obra de Federico Rubio. ProTesis, 2019. Recuperado a partir de http://ciberindex.com/c/proT/pt20091 Santainés Borredá, E., Faus Gabandé, F. \& Camaño Puig, R. (2016). Cuidados de Nightingale en la sala del enfermo y su repercusión en Rubio y Galí. Revista Enfermagem UERJ, 24(2), 17019.

Santainés Borredá, E., Faus Gabandé, F., Camaño Puig, R., Sarturi, F. (2012). Florence Nightingale y Federico Rubio: reformadores de la Enfermería moderna española. Cultura de los Cuidados, XVI (33), 43-49.

Watts, D. (2005). Seis grados de separación. La ciencia de las redes en la era del acceso. [Six degrees. The Science of a Connected Age.] (F. Meler-Ortí Trans.). Barcelona: Ediciones Paidós Ibérica, S.A.

Woodham Smith, C. (1957). Florence Nightingale; la heroína de los hospitales [Florence Nightingale] (L. Echávarri Trans.). Buenos Aires: Losada, S.A.

Yagüe, R. (1924). Don Federico, iniciador precursor. Revista ibero-americana de ciencias médicas, LV (CCXXXV), 129-132. 